\title{
The keys to healthy family child care homes intervention: Study design and rationale
}

\author{
Truls Østbye ${ }^{\mathrm{a}}$, Courtney M. Mann ${ }^{\mathrm{a}, *}$, Amber E. Vaughn ${ }^{\mathrm{b}}$, Rebecca J. Namenek Brouwer ${ }^{\mathrm{a}}$, \\ Sara E. Benjamin Neelon ${ }^{a}$, Derek Hales ${ }^{b}$, Shrikant I. Bangdiwala ${ }^{c}$, Dianne S. Ward ${ }^{\mathrm{b}, \mathrm{d}}$ \\ a Department of Community and Family Medicine, Duke University Medical Center, 2200 W. Main St., Suite 600, Room 621, DUMC Box 104006, Durham, NC 27705, USA \\ b Center for Health Promotion and Disease Prevention, University of North Carolina at Chapel Hill, 1700 Martin L. King Jr. Blvd., CB 7426, Chapel Hill, NC, 27599-7426, USA \\ c Department of Biostatistics, University of North Carolina at Chapel Hill, 137 E Franklin St Ste 203, Campus Box 8030, Chapel Hill, NC 27514, USA \\ d Department of Nutrition, Gillings School of Global Public Health, University of North Carolina at Chapel Hill, 2207 McGavran-Greenberg Hall, CB 7461, Chapel Hill, \\ NC 27599-7461, USA
}

\section{A R T I C L E I N F O}

\section{Article history:}

Received 4 August 2014

Received in revised form 31 October 2014

Accepted 1 November 2014

Available online 8 November 2014

\section{Keywords:}

Overweight

Obesity

Randomized controlled trial

Children

Family child care homes

\begin{abstract}
A B S T R A C T
Background: Obesity is a major public health problem for which early preventive interventions are needed. Large numbers of young children are enrolled in some form of child care program, making these facilities influential environments in children's development. Family child care homes (FCCH) are a specific type of child care in which children are cared for within the provider's own residence. FCCHs serve approximately 1.5 million children in the U.S.; however, research to date has overlooked FCCH providers and their potential to positively influence children's healthrelated behaviors.

Methods: Keys to Healthy Family Child Care Homes (Keys) is a cluster-randomized controlled trial testing the efficacy of an intervention designed to help providers become healthy role models, provide quality food- and physical activity-supportive FCCH environments, and implement effective business practices. The intervention is delivered through workshops, home visits, tailored coaching calls, and educational toolkits. Primary outcomes are child physical activity measured via accelerometry data and dietary intake data collected using direct observation at the FCCH. Secondary outcomes include child body mass index, provider weight-related behaviors, and observed obesogenic environmental characteristics.

Conclusion: Keys is an innovative approach to promoting healthy eating and physical activity in young children. The intervention operates in a novel setting, targets children during a key developmental period, and addresses both provider and child behaviors to synergistically promote health.
\end{abstract}

(c) 2014 Published by Elsevier Inc.

\section{Background}

Combating childhood obesity is a public health priority for which preventive interventions are needed. Although recent data suggests an improving weight profile among preschoolers

\footnotetext{
* Corresponding author. Tel.: +19196813030.

E-mail address: courtney.mann@dm.duke.edu (C.M. Mann).
}

[1], $25 \%$ of these children are still overweight or obese [2]. Being overweight in childhood is associated with a wide range of adverse health outcomes [3-12] and young children who are overweight or obese are more likely to be overweight or obese in adolescence and adulthood [13,14]. This evidence, and recent data indicating that obese school aged children reach that status by age five [15], highlights the necessity for intervention before excess weight becomes an issue and while children are still developing important dietary and activity habits. 
Child care has been identified as an important setting for obesity prevention efforts [16,17]. Many young children in the US are in some type of non-parental care for a large part of each week [18-20]. Research has shown that child care facilities have substantial influence on physical activity levels during the day $[21,22]$ and provide $50 \%$ to $70 \%$ of a child's daily calorie intake [23]. Unfortunately, many preschool-age children fail to accumulate the recommended $90-120$ min a day of active play [22,24-27], and the foods served often do not meet U.S. Department of Agriculture (USDA) guidelines, particularly for vegetables, whole grains, and milk [28]. Further, personal characteristics of program directors, such as Body Mass Index (BMI), have been shown to be associated with program practices, such as servings of canned fruit, whole grains, and low-fat milk [29]. Therefore, the child care setting and the providers themselves are important targets for intervention efforts.

Most child care-based interventions have been programs developed for centers, which may or may not be transferable to other types of child care settings. Family child care homes $(\mathrm{FCCH})^{1}$ are a unique type of child care setting, one that serves approximately 1.5 million children in the US [20]. An FCCH is a small business that operates out of the provider's own home where children are cared for in a more intimate home environment. In $\mathrm{FCCH}$, one provider, who serves as a constant while the children are enrolled in that home, cares for children of all ages. Unfortunately, evidence suggests that time spent in FCCHs during infancy is associated with increased weight at one and three years of age [30], thus making these homes an even more critical target for early childhood obesity prevention efforts.

Keys to Healthy Child Care Homes (Keys) is one of the first studies to develop and evaluate an obesity prevention intervention specifically for FCCHs. This innovative intervention is designed to help FCCH providers foster healthy physical activity and eating habits in young children, and thereby promote healthy growth and weight. This manuscript presents the background, rationale, and design, of the Keys study.

\section{Methods}

\subsection{Formative work}

To inform the development of the Keys study, the team conducted a survey of local FCCH providers, followed by an abbreviated intervention pilot study. The survey was administered in both an online and in paper format and was completed by 89 providers. Of providers responding to the survey, $57.5 \%$ were African American, $40 \%$ were White, and $2.5 \%$ were Asian. Mean age of providers was 46.2 years and $74 \%$ were overweight or obese. While most FCCHs served breakfast (75\%), lunch (81\%), and an afternoon snack (93\%), 35\% of these homes served fewer than three servings of fruit and vegetables per day, $46 \%$ did not serve whole grains, and $49 \%$ served whole rather than reduced fat milk. Additionally, 32\% of homes provided less than one teacher-led physical activity session per day.

\footnotetext{
${ }^{1}$ FCCH: Family Child Care Home.
}

The intervention pilot study included five providers ( $80 \%$ African American, and all with some college education) and 15 children. Baseline measures showed that nearly all providers had high perceived self-efficacy to provide children with physical activity opportunities and healthy meals; however, assessment of FCCH's physical activity and nutrition environments (using a modified version of the Environment Policy Assessment and Observation (EPAO) [31] tool) showed the need for significant improvements. Children spent about $8.4 \mathrm{~h}$ per day in care, $3 \mathrm{~h}$ in non-sleeping sedentary activities, but only 20 min of moderate to vigorous physical activity (MVPA) was accumulated [27]. Healthy Eating Index (HEI) [32] scores, calculated from observed dietary intake [33], indicated that the quality of children's dietary intake needed improvement (mean score $=63.8$, compared to a score of $80 / 100$, which is considered "good" [34]). Furthermore, four of the five providers were classified as overweight or obese.

The 12-week pilot intervention briefly addressed three proposed program components: Healthy You, Healthy Home, and Healthy Business. The condensed format included a selfassessment, a home visit, a workshop, and a series of contacts from the coach (by mail, email, phone, or web). Providers were highly receptive to the pilot content, indicating that it offered useful information and helpful tools to improve their FCCH business, and provided support for healthy physical activity and nutrition behaviors for both themselves and the children in their care. In addition, providers also reported that the measurement burden was acceptable.

From this formative work, the provider survey and brief intervention pilot, our team gained a better understanding of the issues and topics that resonate with $\mathrm{FCCH}$ providers, firsthand knowledge of measuring in the $\mathrm{FCCH}$ environment, and a gauge of the feasibility of conducting a multi-component intervention in this novel setting. It quickly became clear that the healthy business portion and in-person workshops, while always important, were very popular. While some measurement logistics were simpler, dealing with one person instead of 3-5 in a child care center, measurement burden had to be more closely assessed and monitored. Part of the solution was to have observers complete the measurements whenever possible (i.e., recording exact times when children went outside) and separating the provider questionnaires into smaller parts given over a few days. We also found that compared to child care centers, FCCH providers had a closer relationship with parents. This created both additional opportunities and challenges for intervention development. We were also able to evaluate acceptability and preference for intervention delivery, estimate the number of children to expect, evaluate the structure of the day, and identify variations in outside and inside spaces that would need to be considered for intervention and measurement purposes.

\subsection{Study design}

Keys is a two-arm, cluster-randomized controlled trial (RCT) designed to evaluate the efficacy of a nine-month child obesity prevention intervention that promotes (1) providers as healthy role models, (2) physical activity- and nutrition-supportive environments at the $\mathrm{FCCH}$, and (3) healthy business practices. The final sample will include 150 FCCHs and 450 children aged 18 months to 4 years. FCCHs are randomly assigned (1:1) to the 
intervention or control arm. Outcome measures are collected at baseline and follow-up to assess changes in children's physical activity and diet quality at the FCCH (primary outcomes), as well as children's BMI, providers' physical activity and diet quality, and FCCHs' environmental characteristics. Fig. 1 provides an overview of the study design. The study has been approved by the Institutional Review Boards at the University of North Carolina at Chapel Hill and Duke University Medical Center and informed consent was obtained from all participants.

\subsection{Subject recruitment and eligibility}

A convenience sample of $150 \mathrm{FCCHs}$ is being recruited in multiple waves over the course of three years, from counties across central North Carolina (NC). To help target FCCH in highneed areas, recruitment efforts focus primarily on counties with a higher-than-average prevalence of childhood overweight and obesity among children aged two to four years [35] and belowaverage median household income. Within each county, community partners with established relationships with local FCCHs are engaged to help distribute study information via preferred communication channels (e.g., newsletters, emails, partner website, trainings, group meetings). In addition, an invitation

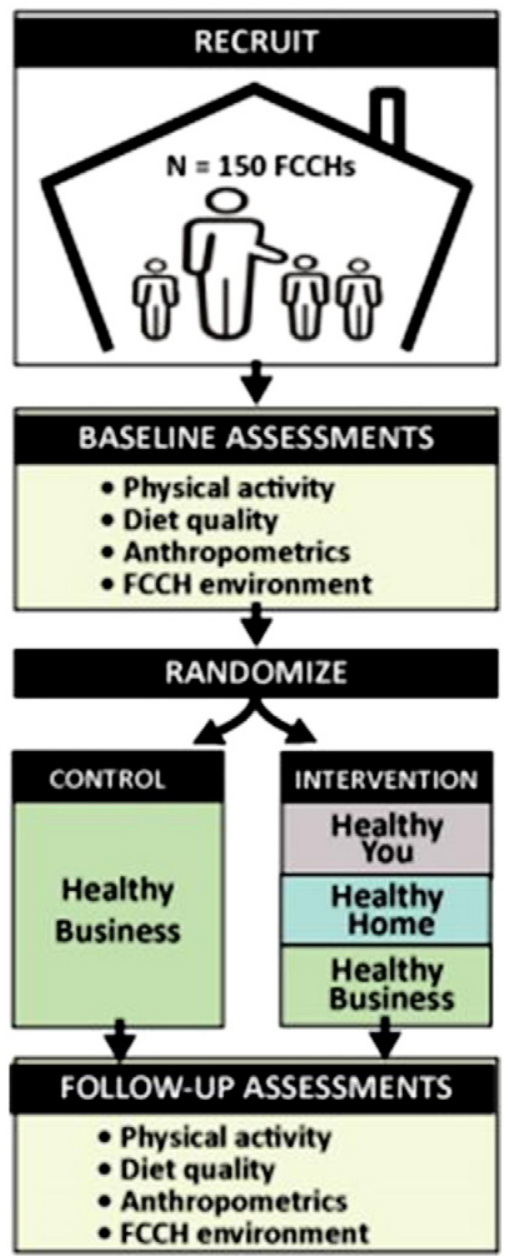

Fig. 1. Study design. to participate in the study and a study flyer are emailed and mailed directly to all FCCHs in the county that are listed in the NC Division of Child Development's publically available database of licensed child care programs. Currently, this database lists about 2350 licensed FCCHs in NC. For each wave, a catchment area with at least 250 FCCHs is identified to ensure adequate recruitment. Invitations are followed by telephone calls from specially trained recruitment staff to review details of study participation and screen for eligibility.

Eligible FCCHs must have at least two children currently enrolled who are between the ages of 18 months and 4 years (but not entering kindergarten before follow-up measures), serve at least one meal and one snack to children, and have been in business for two years (as a demonstration of business stability) with no plans to close in the coming year.

FCCHs that are eligible and interested receive welcome packets. These welcome packets are delivered primarily inperson so that recruitment staff can clearly explain all materials included. The welcome packet contains a provider consent form as well as parent packets. These parent packets provide a brief study overview and a parent consent form for child participation. To remain eligible to participate, the provider must return signed consent for his/her own participation as well as consent from at least two parents with children in the target age group. Providers who care for their own eligible child(ren) may choose to enroll their own child. Once a FCCH is formally enrolled, baseline measures are collected (see Measures below).

These recruitment efforts anticipate that only $10 \%$ of FCCHs invited to participate will end up enrolling in the study. FCCHs who enroll may represent those who are more motivated; however, multiple demographic characteristics of the provider and the FCCH are being monitored (see Measures below) in order to assess the representativeness of the study sample compared to the $\mathrm{FCCH}$ population in our target areas.

\subsection{Randomization}

After all required baseline measurements are collected, the FCCH is stratified into three groups based on the providers' BMI (normal, overweight, obese). While additional stratification variables were considered (e.g., number of children enrolled, enrollment fees, star rating, inclusion of infants), the investigators and statistician agreed to limit stratification to this one variable given the potential for providers' weight to impact their practices and the low prevalence of normal weight among this population. Within each group, FCCHs are randomly assigned to either the intervention or control arm (1:1), using a computerized block randomization approach to ensure equal numbers of each group of FCCHs in both study arms (SAS 9.3 Cary, NC).

\subsection{Intervention}

\subsubsection{Conceptual model}

The Keys intervention is guided by the Socio-Ecologic Framework [36] and components of Social Cognitive Theory [37]. (See Fig. 2 for intervention conceptual model.) The key gatekeeper for the FCCH is the owner/provider; hence the intervention is designed to be delivered to providers who then implement changes that influence the FCCH environment and thereby the behaviors of children cared for in the FCCH. The 


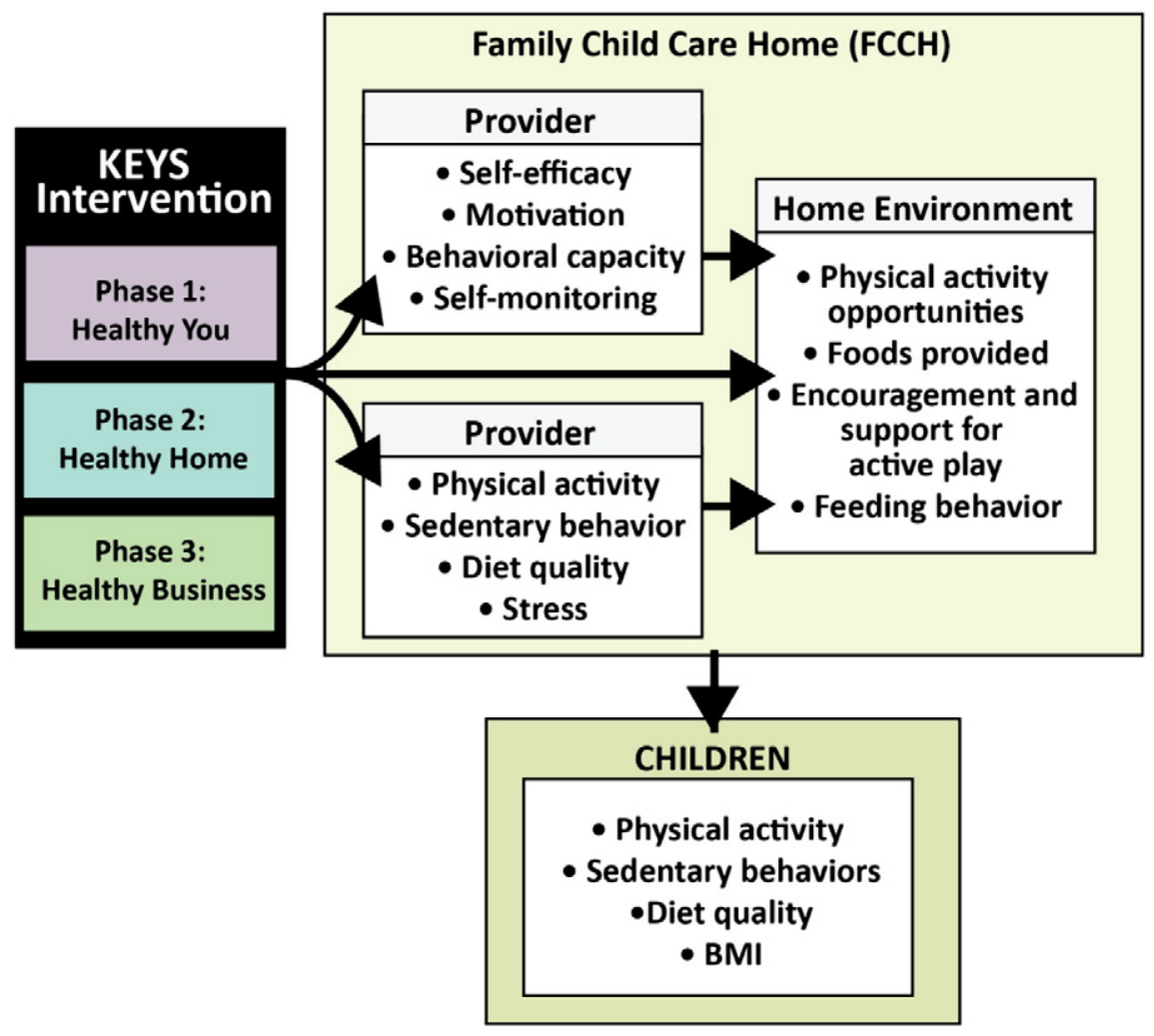

Fig. 2. Conceptual model of Keys intervention.

Socio-Ecologic Framework and its previous application to child obesity prevention [16,38] helped identify several levels of influence to target, specifically: individual (child and provider), interpersonal (interactions between the provider and the child and communication between provider and parents), and environmental (policies, opportunities afforded and limitations imposed by the FCCH). Several behavior change strategies from Social Cognitive Theory [37] were incorporated into intervention design and messages to support providers in making these changes, specifically: goal setting, self-regulation, and selfefficacy. Additionally, intervention delivery incorporates Motivational Interviewing $[39,40]$ informed techniques and adult learning principles [41].

\subsubsection{Content}

The Keys intervention is delivered over nine months, spending approximately three months on each of three modules. These modules are designed to help providers (1) modify their own weight-related behaviors so that they can become role models for children (Module 1: Healthy You), (2) create environments that encourage and support children's physical activity and healthy eating habits (Module 2: Healthy Home), and (3) adopt sound business practices that will help them sustain the changes introduced (Module 3: Healthy Business).

The first module of the intervention, Healthy You, targets the provider's own health behaviors, including their physical activity, sedentary time, dietary intake, eating behaviors, weight, stress, and sleep. Providers are encouraged to compare their current health behaviors against current recommendations and to select priorities and set goals around behaviors they would like to improve. Content not only explains what target behaviors look like, but also provides a variety of simple strategies for how the providers might incorporate these habits into their normal routines. Providers' own health is addressed first as these habits may influence the environment of their FCCH. Healthy providers should be better able to care for children, serve as good role models, and be health advocates for the children in their care.

The second module, Healthy Home, targets the nutrition and physical activity environment of the FCCH. Again, providers are encouraged to compare their current practices against recommendations and to select priorities and set goals around practices they would like to improve. Content in this module helps providers examine multiple aspects of the environment, including the foods and beverages served, the time provided for physical activity and sedentary behaviors, the day-to-day interactions between providers and children around food and physical activity, the physical environment where children eat and play, the communication between providers and parents around efforts to promote healthy living, and the policies that govern these practices.

The final module, Healthy Business, targets the financial health of the $\mathrm{FCCH}$, addressing record keeping for tax purposes, creating policies to minimize the impact of client turnover and payment delinquency, creating and enforcing comprehensive contracts and policies, communicating with parents as customers, professionalism, and marketing. Components of Healthy Business are also integrated into earlier modules to 
help reinforce the financial feasibility and benefits of making changes. Cost is a major barrier to change, especially for $\mathrm{FCCH}$ providers who are self-employed, small business owners in an industry characterized by low profit margins. Helping them improve the operation of their business may help alleviate a real source of stress (lack of financial stability) and increase their self-efficacy for making and sustaining changes both to their own health and to the FCCH environment.

The content from these three modules is intended to work synergistically to encourage children's healthy eating and physical activity habits, and thereby healthy weight. For example, helping providers improve the quality of their diet (e.g., more fruits, vegetables, whole grains, and lean meats, with less sugar sweetened beverages and sweet and salty snacks) should allow them to be healthy role models as these same changes are made to foods served to children. Helping providers improve their personal physical activity behaviors should also help them become more confident in encouraging and leading children in physical activities. Strengthening their business practices should help them identify low-cost, sustainable changes and to use those improvements to enhance the marketing of their FCCH.

Participants in the control arm receive the Healthy Business content as described above. However, this content is divided into three modules (record keeping, contracts and policies, and marketing) and are expanded upon in order to create enough content to mimic the timing and level of attention in the intervention arm (one workshop every three months and four coaching contacts), thus reducing potential attention control bias. The only difference in attention is the replacement of the home visit in each module with a phone call. The focus on business topics is relevant and, therefore, appealing to providers in the control arm.

Materials in the intervention arm related to diet and physical activity for adults and children were written and designed by the study team, a multidisciplinary team of researchers with training and expertise in nutrition and physical activity, related obesogenic behaviors and environments, education, and curriculum development. With the exception of some workshop activities, all materials for Healthy Business (module three of the intervention materials and all control materials) were developed by Tom Copeland (consultant to the study), a lawyer with a business background specializing in business practices specifically for FCCHs. Business related workshop activities were designed to reinforce Tom Copeland's curriculum content and were developed by the intervention manager and health behavior coaches after being trained on Tom Copeland's curriculum and receiving one on one training sessions with Tom Copeland.

\subsubsection{Delivery}

The delivery structure for each of the three modules in the intervention is consistent. First, the FCCH is assigned a dedicated health behavior coach from the study team. Health behavior coaches have backgrounds in counseling related fields, such as social work and counseling, and have been trained in Motivational Interviewing (MI), intervention content, and intervention protocols. The coach delivers: one group workshop, one in-person visit to the $\mathrm{FCCH}$, three tailored coaching phone calls, and an educational toolkit with resources for each of the three modules.
Prior to each group workshop, FCCH providers are asked to complete a self-assessment that helps coaches target potential areas of improvement. Self-assessments are reviewed during the group workshop and the first home visit to help the coach and provider select goals for each module. Details of the intervention delivery model for each module are depicted in Fig. 3.

\subsection{Measures}

\subsubsection{Data collection}

FCCH providers and consented children complete a series of assessments at two time points-baseline and post-intervention. Most of these assessments are collected during a two-day home visit conducted by data collectors, who are trained and certified on all protocols and blinded to randomization. The visits are scheduled over a three-day period (e.g., Monday and

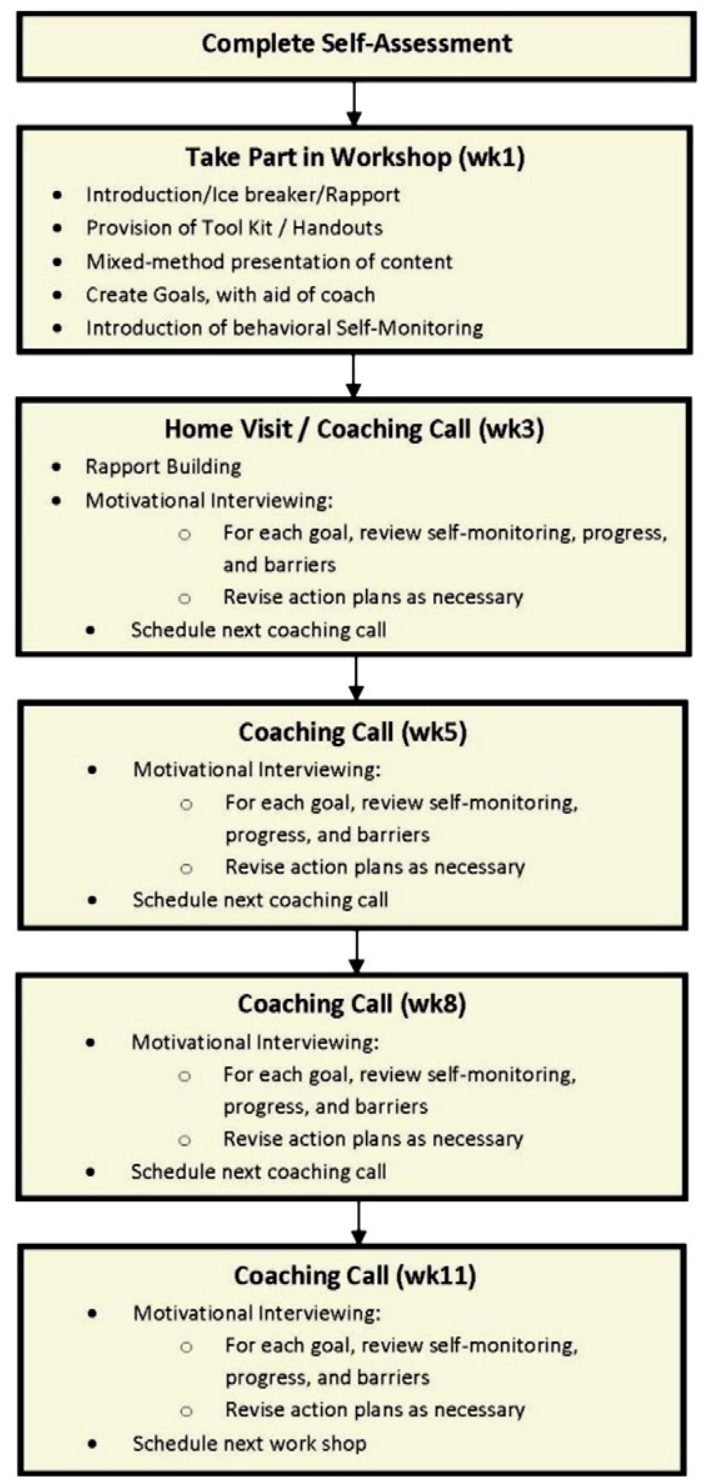

Fig. 3. Delivery model. 
Wednesday or Tuesday and Thursday). The data collectors arrive in the morning before the first meal and stay until the children leave.

\subsubsection{Children's physical activity}

To assess physical activity during child care, children are fitted with an ActiGraph GT3X + accelerometer (ActiGraph, Pensacola, FL). Monitors are worn on a belt over the right hip, for three days. While the primary outcome is for physical activity at child care, children are asked to wear monitors during all waking hours, including those hours when the child is not in the $\mathrm{FCCH}$. Adult caregivers ( $\mathrm{FCCH}$ providers and parents) are instructed about monitor wear so that they can provide assistance and reminders to children. After the home visit, accelerometer data are downloaded and processed to assess non-wear. The most relevant and current algorithms are applied during this process. Minutes of MVPA and sedentary time are estimated by applying established cut points for this age group to the 15-second epoch data files [42-44]. Given slight discrepancies between established cut points, an averaged cut point of less than 25 counts per epoch was used for sedentary (based on Evenson's cut point of 0-25), and 500+ counts per epoch for moderate or vigorous activity (based on Pate's cut point of 420, Evenson's cut point of 574, and Trost's cut point of 418). Estimates for physical activity while at child care are then calculated from full day data by applying start and end times for each child care day.

\subsubsection{Children's diet quality}

Assessment of the children's diet quality is based on observed intakes of foods and beverages consumed while at the FCCH. Using the Diet Observation in Child Care protocol [33], data collectors observe participating children at all meals and snacks served over the course of the day, estimating the amount of foods served, wasted, exchanged, and remaining at the end of the meal or snack. The original protocol was adapted to capture and differentiate foods brought from home. These records are entered into the Nutrition Data System for Research (NDSR, University of Minnesota, Minneapolis-2014) to estimate intakes of energy (kcals), macro- and micronutrients, and servings of different food groups. The HEI $[32,45]$ is then used to assess diet quality based on the comparison of observed intake to that of national dietary guidelines [45].

\subsubsection{Children's anthropometric measures}

To assess children's weight status, data collectors measure the height, weight, and waist circumference of participating children. The procedures are similar to those used in NHANES [46] for this age group. Specifically, height is measured in the standing position (unless the child is not able) to the nearest 1/8 inch using Seca 123 portable stadiometer (Seca Corporation, Columbia, MD); weight measured to the nearest 0.1 pound using a Tanita 800BWB portable scale (Tanita Corporation, Tokyo, Japan); and waist circumference is measured to the nearest $0.1 \mathrm{~cm}$ using a Gulick II measuring tape. Measurements are taken twice and averaged unless they differ by more than a predetermined criterion. In this case a third measure is taken and the closest two are averaged. Height and weight measures are used to calculate body mass index (BMI). Sex-specific growth charts from the Centers for Disease Control and Prevention [47] are then used to calculate BMI percentile and BMI z-score.

\subsubsection{Provider physical activity, diet, and anthropometrics}

Providers' physical activity is assessed over seven days using belt-mounted ActiGraph GT3X + accelerometers worn over the right hip. Monitors are mailed back and data are processed to assess non-wear. Minutes of MVPA and sedentary time are quantified based on cut points established for adults in NHANES [48]. Dietary intakes are assessed with the Block Brief Food Frequency Questionnaire (FFQ) [49]. Comparisons between the longer form (100 items) and the short form (60-item) FFQ support equivalency of the short form [49-51]. Provider height and weight are measured using the same equipment and procedures as described above.

\subsubsection{FCCH environment}

The Environment and Policy Assessment and Observation (EPAO) is used to measure the FCCH's environment and ability to support healthy lifestyles [31]. The EPAO assesses the nutrition and physical activity environments in child care, including the provisions, practices, and policies. While originally developed for use in child care centers, the EPAO was used successfully in our pilot study with only slight modifications. For example, questions about staff behaviors were rephrased to ask about "the provider" instead of "any staff." Questions about the physical space were adjusted to account for the potential lack of a discrete classroom space. For example, when asking about the presence of TV during meals and snacks, a response option was added to capture presence of a TV in an adjacent space that could still be heard. For this study, data collectors completed two days of the EPAO protocol.

\subsubsection{Provider surveys}

To assess potential mediating and moderating variables, providers complete a series of self-administered questionnaires. The demographic questionnaire captures characteristics about the provider (e.g., age, sex, race/ethnicity, household income, education) and the home (e.g., ages of children, race/ethnicity of children, quality rating, and enrollment fees). Motivation to encourage children to eat healthy and be physically active is assessed with a modified version of the Intrinsic Motivation Inventory [52]. Providers' self-efficacy to eat healthy and be physically active is assessed using established scales by Sallis [53] and Kroll [54]. Providers' sleep habits [55,56] and stress [57] are also assessed using established scales. Finally, a modified version of the Business Administration Scale (BAS) [58] is used to capture providers' standard business practices. Modifications to the BAS added items about communication with parents around children's physical activity and eating.

\subsection{Statistical analysis}

For each of the two primary outcomes, physical activity (MVPA) and HEI score, the difference in mean change at followup between intervention and control arms will be compared. The hypotheses are that the children in the intervention arm will increase their MVPA and improve their HEI score more than the children in the control arm. These hypotheses will be tested under the intent-to-treat principle using Generalized Linear Mixed Models (GLMM) that will account for the correlation induced by the clustering of children within FCCHs.

To further explore the effect of the intervention, GLMMs will be fit that (1) adjust for baseline covariates of interest, 
considered a priori, relevant to change in MVPA and Health Eating Index score; (2) adjust for baseline variables distributed differently between intervention groups; (3) test interaction terms between treatment group and other covariates; and (4) examine completers only.

Differences in the secondary outcomes, child BMI, provider weight-related behaviors, and obesogenic FCCH environmental characteristics, will be analyzed in a parallel fashion. Mediating and moderating effects of the factors mentioned above will also be explored.

\subsection{Process evaluation}

A comprehensive process evaluation plan is in place to evaluate intervention implementation, including reach, dose delivered, dose received and fidelity. Reach will be assessed using data collected in the recruitment tracking database. This database records the total number of licensed FCCHs present in the targeted areas, as well as the number screened, not interested, not eligible (along with reasons for ineligibility), consented, and measured. These data will allow us to assess what percent of eligible FCCHs were reached through this study and to explore differences between participants and nonparticipants. Dose delivered, dose received, and fidelity will be monitored using data from the intervention tracking database. This database captures attempts to deliver intervention components (dates of workshops, coaching attempts), completion of each component by participant, and date of completion. These data will allow us to evaluate our delivery of the intended intervention (dose delivered), participant completion rates (dose received), and fidelity to protocol (completion within the specified time frame). In addition, coaching contacts will be recorded and reviewed to assess the use of MI tactics and coverage of intervention topics. Additionally, process evaluation surveys designed to assess provider perceptions of content, implementation methods, and burden of the program will be administered after each module of the intervention.

\subsection{Participant incentives}

FCCH providers are given a variety of incentives for participation in the study. They are offered $\$ 75$ for completion of baseline measures, $\$ 125$ for completion of follow up measures, as well as Continuing Education Units (CEU) for participation in the intervention or control program. As part of both the intervention and control programs, participants receive educational toolkits with incentives such as pedometers, exercise bands, reusable water cups, cork boards, shopping pads, file boxes, and books for their FCCH. The intervention group also gets divided plates and polyspots to use with the children in their homes. All of these items are designed to be useful to the FCCH while also supporting program participation. Additionally, the business content of the intervention is designed to be an incentive to participate.

\section{Discussion}

The Keys study is one of the first to evaluate a child obesity prevention intervention designed specifically for use in FCCHs. A recent review of obesity prevention interventions in children from birth to age five identified 50 trials, none targeting FCCHs specifically [59]. Since that review, Trost et al. published results from their Healthy Kansas Kids intervention, which trained local community Resource and Referral Agencies to provide a series of eight technical assistance visits to family child care home providers to help them improve their policies and practices related to healthy eating and physical activity [60]. In addition, de Silva-Sanigorski et al. published results from the implementation of the community-based Romp and Chomp intervention on family day care programs. [61] Results from both of these studies suggest that family child care settings are amenable to obesity prevention intervention efforts. The remainder of the research that has been done with FCCHs has been crosssectional studies focuses on characterizing their physical activity or nutrition environments and practices [62-70]. Some of these studies suggest that children in FCCHs get only 14 min of MVPA per 8-hour day [64] and watch significantly more television compared to those in center-based care $[69,70]$. There is also some evidence that $56 \%$ of family child care homes are serving children multiple servings of juice each day and $86 \%$ are serving higher fat milk [62]. Additionally, less than $50 \%$ of providers in $\mathrm{FCCHs}$ receive annual training on physical activity or nutrition [62]. More research in FCCHs is needed; however, the little evidence that is available suggests that FCCHs are an important target for obesity prevention that are responsive to interventions.

The Keys intervention is unique among child care-based interventions to date in that it recognizes the importance of not only the providers' personal health habits and their business practices but also the potential impact these factors have on their ability to implement and sustain changes in support of children's healthy behaviors. Few, if any, child care-based obesity prevention programs have addressed provider health, yet the providers are key gatekeepers whose personal health and habits impact the nutrition and physical activity opportunities offered to children in their care $[63,71,72]$. Furthermore, few obesity prevention programs have incorporated messages around economic stability. Such an approach is necessary when trying to implement and sustain changes in a business characterized by low profit margins and should allow more resources and attention to be paid to the creation of a healthy, non-obesogenic environment. To address these issues, the Keys intervention includes three separate modules-Healthy You, Healthy Home, Healthy Business-which are designed to work together synergistically to create $\mathrm{FCCH}$ environments that foster healthy eating and physical activity habits in children.

The use of coaches to provide tailored technical assistance to the FCCH providers throughout each phase is also novel. Although coaches have been used in other health behavior interventions, no studies have employed such an approach to simultaneously modify organizational policies, environments, and practices. In Keys, the role of the health behavior coach includes reinforcement of key messages about FCCH business "health" and viability, as well as physical activity- and nutritionrelated environmental support for the children.

FCCHs are important settings through which healthy weight-related behaviors for young children can be promoted. However, these settings have special considerations that must be addressed when designing obesity prevention intervention studies. FCCHs are small businesses, with low profit margins, where providers are caring for children across a variety of age groups simultaneously. Change strategies must be low-cost, easy 
to implement and sustain, and applicable to infants, toddlers, and preschoolers. FCCH providers usually have a close relationship with both the children in their care and their parents; hence they can become powerful role models of healthy behaviors and advocates of healthy practices. By supplying the necessary knowledge, tools, and motivational coaching, the Keys to Healthy Homes program should be an effective tool for improving the diet quality and increasing the physical activity of children in FCCHs.

\section{Acknowledgments}

We would like to thank the following people who contributed to the development and execution of the Keys project:

Temitope Erihosho, Tracy Itty, Joy Long, Sakinah Omar, Rachel Bartlett, Andrew DeGraff, Tom Copeland, and our Community Advisory Group of local family child care home providers.

This research was funded by the National Heart, Lung, and Blood Institute of the National Institutes of Health under Award Number R01HL108390. The content is solely the responsibility of the authors and does not necessarily represent the official views of the National Institutes of Health. The sponsor had no role in the study design; collection, analysis, or interpretation of the data; writing of the article; or decision to publish this article.

\section{References}

[1] Ogden CL, Carroll MD, Kit BK, Flegal KM. Prevalence of childhood and adult obesity in the United States, 2011-2012. JAMA 2014;311(8):806-14.

[2] Ogden CL, Carroll MD, Kit BK, Flegal KM. Prevalence of obesity and trends in body mass index among US children and adolescents, 1999-2010. JAMA 2012;307(5):483-90.

[3] Freedman DS, Dietz WH, Srinivasan SR, Berenson GS. The relation of overweight to cardiovascular risk factors among children and adolescents: the Bogalusa Heart Study. Pediatrics 1999;103(6 Pt 1):1175-82.

[4] Roberts EA. Pediatric nonalcoholic fatty liver disease (NAFLD): a "growing" problem? J Hepatol Jun 2007;46(6):1133-42.

[5] Rodriguez MA, Winkleby MA, Ahn D, Sundquist J, Kraemer HC. Identification of population subgroups of children and adolescents with high asthma prevalence: findings from the Third National Health and Nutrition Examination Survey. Arch Pediatr Adolesc Med Mar 2002;156(3):269-75.

[6] Sinha R, Fisch G, Teague B, Tamborlane WV, Banyas B, Allen K, et al. Prevalence of impaired glucose tolerance among children and adolescents with marked obesity. N Engl J Med 2002;346(11):802-10.

[7] Steinberger J, Moran A, Hong CP, Jacobs Jr DR, Sinaiko AR. Adiposity in childhood predicts obesity and insulin resistance in young adulthood. J Pediatr Apr 2001;138(4):469-73.

[8] Weiss R, Dziura J, Burgert TS, Tamborlane WV, Taksali SE, Yeckel CW, et al. Obesity and the metabolic syndrome in children and adolescents. N Engl J Med 2004;350(23):2362-74.

[9] Erickson SJ, Robinson TN, Haydel KF, Killen JD. Are overweight children unhappy?: Body mass index, depressive symptoms, and overweight concerns in elementary school children. Arch Pediatr Adolesc Med Sep 2000;154(9):931-5.

[10] Duarte CS, Sourander A, Nikolakaros G, Pihlaiamaki H, Helenius H, Piha J, et al. Child mental health problems and obesity in early adulthood. J Pediatr Jan 2010;156(1):93-7.

[11] Wang F, Wild TC, Kipp W, Kuhle S, Veugelers PJ. The influence of childhood obesity on the development of self-esteem. Health Rep Jun 2009;20(2): 21-7.

[12] BeLue R, Francis LA, Colaco B. Mental health problems and overweight in a nationally representative sample of adolescents: effects of race and ethnicity. Pediatrics Feb 2009;123(2):697-702.

[13] Baird J, Fisher D, Lucas P, Kleijnen J, Roberts H, Law C. Being big or growing fast: systematic review of size and growth in infancy and later obesity. BMJ Oct 22 2005;331(7522):929.
[14] Monteiro PO, Victora CG. Rapid growth in infancy and childhood and obesity in later life-a systematic review. Obes Rev May 2005;6(2): $143-54$

[15] Cunningham SA, Kramer MR, Narayan KMV. Incidence of childhood obesity in the United States. N Engl J Med 2014;370(5):403-11.

[16] Birch LL, Ventura AK. Preventing childhood obesity: what works? (2005) Int J Obes (Lond) Apr 2009;33(Suppl. 1):S74-81.

[17] Larson N, Ward DS, Neelon SB, Story M. What role can child-care settings play in obesity prevention? A review of the evidence and call for research efforts. J Am Diet Assoc 2011;111(9):1343-62.

[18] Flynn MA, McNeil DA, Maloff B, Mutasingwa D, Wu M, Ford C, et al. Reducing obesity and related chronic disease risk in children and youth: a synthesis of evidence with 'best practice' recommendations. Obes Rev Feb 2006;7(Suppl. 1):7-66.

[19] Iruka I, Carver P. Initial results from the 2005 NEHS Early Childhood Program Participation Survey. United States Department of Education; 2006 [NCES 2006075].

[20] Laughlin L. Who's minding the kids? Child care arrangements: spring 2011. Washington, DC: U.S. Census Bureau; 2013.

[21] Finn K, Johannsen N, Specker B. Factors associated with physical activity in preschool children. J Pediatr Jan 2002;140(1):81-5.

[22] Pate RR, Pfeiffer KA, Trost SG, Ziegler P, Dowda M. Physical activity among children attending preschools. Pediatrics Nov 2004;114(5): 1258-63.

[23] United States. Department of Agriculture. Food and Consumer Service. Office of Analysis and Evaluation. Early Childhood and Child Care Study: Final report: vol. II. Nutritional assessment of the CACFP. Cambridge, MA: Abt Associates; 1997. Retrieved from http://www.abtassoc.com/reports/ D19971210.pdf.

[24] American Academy of Pediatrics, American Public Health Association. National Resource Center for Health and Safety in Child Care and Early Education3rd ed. ; 2010 [http://nrckids.org/CFOC3/PDFVersion/preventing obesity.pdf].

[25] Reilly JJ. Low levels of objectively measured physical activity in preschoolers in child care. Med Sci Sports Exerc Mar 2010;42(3):502-7.

[26] Reilly JJ, Kelly L, Montgomery C, Williamson A, Fisher Al, McColl JH, et al. Physical activity to prevent obesity in young children: cluster randomised controlled trial. BMJ Nov 18 2006;333(7577):1041.

[27] Pate RR, Mclver K, Dowda M, Brown WH, Addy C. Directly observed physical activity levels in preschool children. J Sch Health Aug 2008;78(8): 438-44.

[28] Ball SC, Benjamin SE, Ward DS. Dietary intakes in North Carolina child-care centers: are children meeting current recommendations? J Am Diet Assoc Apr 2008;108(4):718-21.

[29] Mazzucca SHD, Tabak RG, Vaughn A, Ward DS. Are center or center director characteristics related to diet quality at childcare? Minneapolis, MN: International Society of Behavioral Nutrition and Physical Activity; 2010.

[30] Benjamin SE, Rifas-Shiman SL, Taveras EM, Haines J, Finkelstein J, Kleinman K, et al. Early child care and adiposity at ages 1 and 3 years. Pediatrics Aug 2009;124(2):555-62.

[31] Ward DS, Hales D, Haverly K, Marks J, Benjamin S, Ball S, et al. An instrument to assess the obesogenic environment of child care centers. Am J Health Behav 2008;32(4):380-6.

[32] Kennedy ET, Ohls J, Carlson S, Fleming K. The Healthy Eating Index: design and applications. J Am Diet Assoc Oct 1995;95(10):1103-8.

[33] Ball SC, Benjamin SE, Ward DS. Development and reliability of an observation method to assess food intake of young children in child care. J Am Diet Assoc Apr 2007;107(4):656-61.

[34] Basiotis PP, Carlson A, Gerrior SA, Juan WY, Lino M. The Healthy Eating Index: 1999-2000. U.S. Department of Agriculture, Center for Nutrition Policy and Promotion; 2002.

[35] Nutrition Services Branch, North Carolina Division of Public Health. Prevalence of obesity, overweight, healthy weight and underweight in children 2 through 4 years of age, by county, NC-NPASS. http://www. eatsmartmovemorenc.com/Data/Texts/NCNPASS\%202009\%20County\%2024\%20Years.pdf; 2009. [Accessed June 8, 2011].

[36] McLeroy K, Bibeau K, Steckler A, Glanz K. An ecological perspective on health promotion programs. Health Educ Q 1988;15:351-77.

[37] Bandura A. Social foundations of thought and action. A social cognitive theory. Englewood Cliffs, NJ: Prentice-Hall; 1986.

[38] Davison KK, Birch LL. Childhood overweight: a contextual model and recommendations for future research. Obes Rev Aug 2001;2(3):159-71.

[39] Miller WR, Rollnick S. Motivational Interviewing: preparing people for change. New York: Guilford Press; 2002.

[40] Rollnick S, Miller WR, Butler C. Motivational interviewing in health care: helping patients change behavior. New York: Guilford Press; 2008.

[41] Dunst CJ, Trivette CM, Hamby DW. Meta-analysis of the effectiveness of four adult learning methods and strategies. Int J Continuing Educ Lifelong Learn 2010;3(1):91-112. 
[42] Pate RR, Almeida MJ, Mclver KL, Pfeiffer KA, Dowda M. Validation and calibration of an accelerometer in preschool children. Obesity (Silver Spring) Nov 2006;14(11):2000-6.

[43] Evenson KR, Catellier DJ, Gill K, Ondrak KS, McMurray RG. Calibration of two objective measures of physical activity for children. J Sports Sci Dec 2008;26(14):1557-65.

[44] Trost SG, Fees BS, Haar SJ, Murray AD, Crowe LK. Identification and validity of accelerometer cut-points for toddlers. Obesity (Silver Spring) Nov 2012; 20(11):2317-9.

[45] Guenther PM, Reedy J, Krebs-Smith SM, Reeve BB. Evaluation of the Healthy Eating Index-2005. J Am Diet Assoc Nov 2008;108(11): 1854-64.

[46] Troiano RP, Berrigan D, Dodd KW, Masse LC, Tilert T, McDowell M. Physical activity in the United States measured by accelerometer. Med Sci Sports Exerc Jan 2008;40(1):181-8

[47] Kuczmarski RJ, Ogden CL, Grummer-Strawn LM, Flegal KM, Guo SS, Wei R, et al. Advance data from vital and health statistics; no. 314. National Center for Health Statistics.: Hyattsville, Maryland; 2000

[48] Troiano RP. Translating accelerometer counts into energy expenditure: advancing the quest. J Appl Physiol 2006;100(4):1107-8.

[49] Block GHA, Naughton D. A reduced dietary questionnaire: development and validation. Epidemiology 1990;1:58-64.

[50] Potischman N, Carroll RJ, Iturria SJ, Mittl B, Curtin J, Thompson FE, et al. Comparison of the 60 - and 100-item NCI-block questionnaires with validation data. Nutr Cancer 1999;34(1):70-5.

[51] Subar AF, Thompson FE, Kipnis V, Midthune D, Hurwitz P, McNut S, et al. Comparative validation of the Block, Willett, and National Cancer Institute food frequency questionnaires: the Eating at America's Table Study. Am J Epidemiol 2001;154(12):1089-99.

[52] McAuley E, Duncan T, Tammen VV. Psychometric properties of the Intrinsic Motivation Inventory in a competitive sport setting: a confirmatory factor analysis. Res Q Exerc Sport Mar 1989;60(1):48-58.

[53] Sallis JF, Pinski RB, Grossman RM, Patterson TL, Nader PR. The development of self-efficacy scales for health related diet and exercise behaviors. Health Educ Res September 1, 1988;3(3):283-92.

[54] Kroll T, Kehn M, Ho P-S, Groah S. The SCI Exercise Self-Efficacy Scale (ESES): development and psychometric properties. Int J Behav Nutr Phys Act 2007;4(1):34.

[55] Hays RD, Stewart AL. Sleep measures. Measuring functioning and wellbeing: The Medical Outcomes Study approach; 1992 235-59.

[56] Hays RD, Martin SA, Sesti AM, Spritzer KL. Psychometric properties of the Medical Outcomes Study Sleep measure. Sleep Med Jan 2005;6(1): 41-4.

[57] Sheldon C, Kamarck T, Mermelstein R. A global measure of perceived stress. J Health Soc Behav 1983;24(4):385-96.
[58] Talan TNB, Jorde Paula. Business Administration Scale for Family Child Care, BAS. New York, NY: Teachers College Press; 2009.

[59] Hesketh KD, Campbell KJ. Interventions to prevent obesity in 0-5 year olds: an updated systematic review of the literature. Obesity (Silver Spring) Feb 2010;18(Suppl. 1):S27-35.

[60] Trost SG, Messner L, Fitzgerald K, Roths B. A nutrition and physical activity intervention for family child care homes. Am J Prev Med 2011;41(4): 392-8.

[61] de Silva-Sanigorski A, Elea D, Bell C, Kremer P, Carpenter L, Nichols M, et al. Obesity prevention in the family day care setting: impact of the Romp \& Chomp intervention on opportunities for children's physical activity and healthy eating. Child Care Health Dev May 2011;37(3): 385-93.

[62] Trost SG, Messner L, Fitzgerald K, Roths B. Nutrition and physical activity policies and practices in family child care homes. Am J Prev Med Dec 2009; 37(6):537-40.

[63] Fees B, Trost S, Bopp M, Dzewaltowski DA. Physical activity programming in family child care homes: providers' perceptions of practices and barriers. J Nutr Educ Behav Jul-Aug 2009;41(4):268-73.

[64] Temple VA, Naylor PJ, Rhodes RE, Higgins JW. Physical activity of children in family child care. Appl Physiol Nutr Metab Aug 2009;34(4):794-8.

[65] Monsivais P, Kirkpatrick S, Johnson DB. More nutritious food is served in child-care homes receiving higher federal food subsidies. J Am Diet Assoc May 2011;111(5):721-6.

[66] Gunter KB, Rice KR, Ward DS, Trost SG. Factors associated with physical activity in children attending family child care homes. Prev Med Feb 2012; 54(2):131-3.

[67] Rice KR. Physical activity in children attending family child care homes. [Doctoral Dissertation] Oregon State University; 2012.

[68] Natale R, Page M, Sanders L. Nutrition and physical activity practices in childcare centers versus family childcare homes. Early Child Educ J 2013/ 08/10;2013:1-8.

[69] Christakis DA, Garrison MM. Preschool-aged children's television viewing in child care settings. Pediatrics Dec 2009;124(6):1627-32.

[70] Tandon PS, Zhou C, Lozano P, Christakis DA. Preschoolers' total daily screen time at home and by type of child care. J Pediatr Feb 2011;158(2): 297-300.

[71] Copeland KA, Kendeigh CA, Saelens BE, Kalkwarf HJ, Sherman SN. Physical activity in child-care centers: do teachers hold the key to the playground? Health Educ Res Feb 2012;27(1):81-100.

[72] Hughes CC, Gooze RA, Finkelstein DM, Whitaker RC. Barriers to obesity prevention in Head Start. Health Aff (Millwood) Mar-Apr 2010;29(3): 454-62. 